\title{
Clinical physiology and sleep: insights from the European Respiratory Society Congress 2017
}

\author{
Isaac Almendros ${ }^{1,2,3}$, Andrea Crespo ${ }^{4}$, Olga Tura-Ceide ${ }^{2,3,5}$, Maria R. Bonsignore ${ }^{6,7}$ \\ ${ }^{1}$ Unitat de Biofísica i Bioenginyeria, Facultat de Medicina i Ciències de la Salut, Universitat de Barcelona, Barcelona, Spain; ${ }^{2}$ Centro de Investigación \\ Biomédica en Red de Enfermedades Respiratorias, 28029-Madrid, Spain; ${ }^{3}$ Institut d'Investigacions Biomèdiques August Pi i Sunyer (IDIBAPS), \\ Barcelona, Spain; ${ }^{4}$ Unidad de Sueño. Hospital Universitario Rio Hortega de Valladolid, Valladolid, Spain; ${ }^{5}$ Department of Pulmonary Medicine, \\ Hospital Clinic, Barcelona, Spain; ${ }^{6}$ Biomedical Department of Internal and Specialistic Medicine (DiBiMIS), University of Palermo, Palermo, Italy; \\ ${ }^{7}$ Institute of Biomedicine and Molecular Immunology (IBIM), National Research Council (CNR), Palermo, Italy \\ Correspondence to: Dr. Isaac Almendros. Unitat de Biofísica i Bioenginyeria, Facultat de Medicina i Ciències de la Salut, Universitat de Barcelona, \\ Casanova 143, 08036 Barcelona, Spain. Email: isaac.almendros@ub.edu.
}

Submitted Aug 10, 2017. Accepted for publication Oct 09, 2017.

doi: $10.21037 /$ jtd.2017.11.44

View this article at: http://dx.doi.org/10.21037/jtd.2017.11.44

The 2017 Annual Congress of the European Respiratory Society (ERS) held in Milan has featured the latest research on clinical physiology and sleep. New exciting studies based on respiratory physiology, sleep apnea and its cardiovascular, metabolic and neurocognitive consequences have been presented. Every year, the ERS Congress provides an excellent opportunity to discover the novel insights from basic, translational and clinical studies on Respiratory Medicine. In this review, we highlight some studies addressing: (I) the relationship between sleep apnea and cancer, and (II) the potential benefits of exercise in respiratory disorders.

\section{Update from the European Sleep Apnea Database (ESADA) on cancer-obstructive sleep apnea (OSA)}

Experimental and clinical studies have suggested a potential relationship between OSA and cancer development or progression $(1,2)$. Epidemiological evidence from relatively small cohorts suggests that OSA is associated with higher cancer incidence and mortality, mainly in patients with severe sleep-disordered breathing. In addition, cancer remains one of the main causes of mortality (3). The high epidemiologic impact of both cancer and sleep apnea and the potential for clinical treatment make this field of research an exciting challenge, and more prospective studies are necessary (1). Data from the Busselton Health Study cohort showed that moderate-to-severe sleep apnea is independently associated with a large increase in risk for all-cause mortality, incident stroke, and cancer incidence and mortality (4). In this context, the aim of the study presented by Pataka et al. was to explore the association between the severity of OSA and prevalence of cancer in patients of ESADA after controlling for several known risk factors for cancer development (5). ESADA is a prospective multicenter cohort study of adult patients with OSA defined as an apnea/hypopnea index (AHI) $\geq 5$. OSA severity was classified according to AHI, oxygen desaturation index (ODI) and \% night-time spent with oxygen saturation $<90 \%$ (TSat90). The cross-sectional association between OSA and cancer prevalence was assessed at the time of the baseline visit using logistic regression analysis. There were a total of 19,556 analyzed participants. A total of 357 patients had been diagnosed with malignancy $(1.8 \%)$ and the $59.4 \%$ were male. Patients with malignancy were older $(\mathrm{P}<0.001)$, had lower body mass index $(\mathrm{BMI})(\mathrm{P}=0.004)$ and neck circumference $(\mathrm{P}=0.001)$. There were no differences in the AHI, ODI, TSat90, Epworth Sleepiness Scale (ESS), subjective sleep length and latency, between patients with/ without malignancy. Total sleep time was lower in patients with malignancy $(\mathrm{P}=0.02)$. The multivariate regression analysis found that OSA severity assessed by AHI or ODI was not significantly associated with cancer prevalence after adjustment for age, sex, BMI, smoking status and alcohol intake. However, in patients aged $<50$ years AHI $\geq 5$ was associated with increased risk of cancer $(\mathrm{OR}=2.47$; $95 \% \mathrm{CI}$, 1.13-5.44; $\mathrm{P}=0.024)$. 
These results are in line with those obtained in a multicenter study in consecutive patients investigated for suspected OSA (6). OSA severity was measured by the AHI and the hypoxemia index (TSat90). The closest association with cancer was found in patients $<65$ years for both the AHI (continuous log-transformed AHI: HR $=1.87 ; 95 \%$ CI, 1.1-3.2; upper vs. lower AHI tertile: HR =3.98; 95\% CI, 1.14-3.64) and the TSat90. The conclusion was that OSA severity is associated with increased cancer mortality, particularly in patients aged $<65$ years.

In the same way, Campos-Rodriguez et al. have designed a multicenter, clinical cohort study including consecutive patients investigated for suspected OSA between 2003 and 2007 in seven Spanish teaching hospitals (7). AHI and TSat90 were used as surrogates of OSA severity, both as continuous variables and categorized by tertiles. Increased overnight hypoxia as a surrogate of OSA severity was associated with increased cancer incidence. This association seems to be limited to men and patients younger than 65 years of age. In stratified analyses, TSat 90 was associated with cancer incidence in patients younger than 65 years [adjusted HR $=1.13$ (95\% CI, 1.06-1.21) per 10-unit increase in TSat90] and males [adjusted HR $=1.11$ (95\% CI, 1.04-1.17) per 10-unit increase in TSat90]. As a result, AHI was not associated with cancer incidence in the adjusted analyses, except for patients younger than 65 years (adjusted $\mathrm{HR}$ for $\mathrm{AHI}>43$ vs. $<18.7=1.66$; 95\% CI, 1.04-2.64).

The data from the large multicentric ESADA cohort confirm the association between OSA (AHI cutoff 5) and a prevalent cancer diagnosis, but only in patients aged below 50 years as other authors have shown in previous multicenter studies.

\section{Spontaneous tumorigenesis induced by intermittent hypoxia in aged mice: physiological parameters}

OSA is a very common breath disturbance in older people (20-26\% incidence in age $>70$ years). Recently, several studies have demonstrated that patients with OSA have a higher prevalence of cancer and cancer-related mortality $(4,6)$. The average age of cancer diagnosis is 66 years with $25 \%$ of new diagnosis between $65-74$ years. Human and animal data support the relationship between OSA and cancer, but how spontaneous tumorigenesis in elderly OSA patients affects several physiological parameters has not been investigated. Various pathophysiologic pathways have been postulated as possible causes of cancer or its increased aggressiveness, and also of greater resistance to antitumoral treatment in the presence of both intermittent hypoxia (IH) and sleep fragmentation (both inherent to sleep apnea). Thus far, these biological hypotheses have been supported by various experimental studies in animals $(8,9)$.

Apneic episodes lead to recurrent hypoxemiareoxygenation cycles with concomitant cellular $\mathrm{IH}$, similar to ischemia-reperfusion injury. Studies suggest that IH in OSA may influence tumorigenesis (10). Animal studies indicate that the activation of a subunit of a heterodimeric transcription factor hypoxia-inducible factor 1 (HIF-1) and the vascular endothelial growth factor (VEGF) pathways in response to IH may promote the blood supply which supports tumor growth (11). In addition, tumor-associated macrophages may be altered by IH (or sleep fragmentation) to a tumor-promoting phenotype yielding more aggressive cancer behavior (8).

In this context, the study by Obeso et al. used chronic intermittent hypoxia $(\mathrm{CIH})$, the main consequence of OSA, as the only variable in old mice (12). Two hundred outbred 15 -month-old mice were randomly distributed into three groups: room air, mild $\mathrm{CIH}$ (nadir $12 \% \mathrm{O}_{2}$ ), and severe $\mathrm{CIH}$ (nadir $7.5 \% \mathrm{O}_{2}$ ). The experimental protocol was 30 cycles of $\mathrm{CIH} / \mathrm{h}, 8 \mathrm{~h} /$ day along 3 months, equivalent in human years to 50-60 years of age with 8-10 years of OSA exposure. Whole body plethysmography and blood biochemical parameters were measured immediately before the $\mathrm{CIH}$ exposure and after 6 and 12 weeks of treatment. Mice were sacrificed at the age of 18 months, blood and tissue samples were collected and tumor masses were carefully sought.

There was a decrease in ventilatory parameters due to age, which was attenuated with CIH. Blood glucose, lactate, and cholesterol levels were maintained with age, but glycaemia and lactatemia decreased in CIH mice. Blood triglycerides concentration decreased with age. Abnormal values appeared in mice with tumors. CIH tended to reduce visceral fat. There were no statistical differences between healthy mice and mice with tumors in any of the three groups. Consequently, age seems to protect against the harmful effects of $\mathrm{CIH}$ regardless of spontaneous tumorigenesis.

In this context, Quintero et al. have also shown that age protects from the harmful effects of CIH (13). Since OSA is clinically less conspicuous in aged patients, they compared CIH effects in young (3-4 months) and aged (22-24 months) rats. To define potential distinctive patterns of these pathogenic mechanisms, mean arterial blood pressure was measured as the main outcome of $\mathrm{CIH}$ exposure. In young 
rats, $\mathrm{CIH}$ augmented carotid body (CB) sensory responses to hypoxia, decreased hypoxic ventilation and augmented sympathetic activity (plasma catecholamine levels and renal artery content and synthesis rate). An increased brainstem integration of $\mathrm{CB}$ sensory input as a trigger of sympathetic activity was suggested. $\mathrm{CIH}$ also caused an oxidative status decreasing the aconitase/fumarase ratio and superoxide dismutase activity. In aged animals, CIH minimally affected $\mathrm{CB}$ responses, ventilation and sympathetic-related parameters, leaving redox status unaltered. In young animals, CIH caused hypertension and in aged animals, whose baseline blood pressure was augmented, CIH did not augment it further.

\section{Endurance exercise training and skeletal muscle oxidative capacity in chronic obstructive pulmonary disease (COPD) are linked by genetic variants}

COPD presents several extrapulmonary manifestations, including cachexia and skeletal muscle dysfunction, contributing to mortality. Skeletal muscle dysfunction occurs in all stages of COPD and is characterized by significant loss of muscle strength and endurance, together with a loss of skeletal muscle oxidative capacity (14). Leg muscle dysfunction significantly influences symptoms and health related quality of life, and exacerbates dyspnea and morbidity. However, in contrast to the lungs, alterations in skeletal muscles are potentially be reversed by exercise training (15). A large fraction of variance in aerobic capacity gained by exercise training is accounted for by 11 single nucleotide polymorphisms (16). Taking into account this information, Rossiter et al. hypothesized that the 11 single nucleotide polymorphisms (SNPs) previously associated with responsiveness to training could be associated with low muscle oxidative capacity in COPD (17). Calf oxidative capacity was assessed at rest from the $\mathrm{O}_{2}$ consumption recovery rate constant $(\mathrm{k})$ measured by near-infrared spectroscopy in 195 participants, 90 of them with COPD (GOLD 1-4, n=28/34/18/10). Genetic association analyses were adjusted for age, weight, FEV1\% pred and physical activity (triaxial accelerometry). Results showed that COPD patients had lower oxidative capacity $(\mathrm{k})\left(1.27 \pm 0.39 \mathrm{~min}^{-1}\right)$ than controls $\left(1.68 \pm 0.49 \mathrm{~min}^{-1}\right)$. The authors concluded that specific genetic variants associated with traininginduced gain in aerobic capacity may be associated with low muscle oxidative capacity in COPD. In particular, DEPDC6 appear to be associated with myofiber switch from oxidative to glycolytic metabolism, and TTN (titin) in muscle passive stiffness and elasticity. Although larger populations and mechanistic studies are needed to further confirm these findings, these results indicate that at least part of the heterogeneity in adaptation to exercise therapy might originate from variation in gene sequence influencing the complex biological networks mediating the response to aerobic exercise training. Identification of individuals with a particular risk profile is the first step toward the implementation of personalized medicine, providing a significant new milestone in the field.

\section{Reduced voluntary activation and increased intracortical inhibition in OSA patients}

Reduced muscle strength, endurance with structural and bioenergetic changes have been reported in the skeletal muscle of severe OSA patients (18). Very recently, the severity of OSA was found to be associated with an increased skeletal muscle mass index and decreased skeletal muscle density (19). Higher intracortical inhibition was previously reported at rest in moderate-to-severe OSA by transcranial magnetic stimulation, suggesting central neuromuscular impairment in these patients (20).

The work presented by Marillier et al. investigated the neuromuscular mechanisms of exercise-induced fatigue in severe OSA patients before and after an 8-week continuous positive airway pressure (CPAP) treatment (21). Twelve OSA patients and 11 matched controls were studied. Time to exhaustion was $\sim 30 \%$ lower $(\mathrm{P}=0.017)$ in OSA patients compared to controls. Voluntary activation levels assessed by femoral nerve electrical stimulation (VAFNES) $(86 \% \pm 10 \%$ vs. $89 \% \pm 8 \%, \mathrm{P}=0.022)$ and voluntary activation levels assessed by transcranial magnetic stimulation (VATMS) $(86 \% \pm 16 \%$ vs. $91 \% \pm 7 \%, \mathrm{P}=0.049)$ were lower in OSA patients before and during repeated isometric knee extensions. Based on these results, OSA patients may have reduced knee extensors strength and endurance due to central activation deficit and increased intracortical inhibition. After 8-week of CPAP treatment, the neuromuscular impairments were not reversed. Several studies have shown a favorable effect of supervised exercise training in OSA patients, reducing the severity of the disease and improving cardiorespiratory fitness, daytime sleepiness, and sleep efficiency. This study emphasizes the potential value of exercise in the management of OSA to sustain muscle strength and endurance. Also, it is important to consider that exercise not only can improve OSA, but 
decreased the severity of central sleep apnea in chronic heart failure patients $(22,23)$. Therefore, exercise-training could be helpful to mitigate the cardiovascular disease, impaired glucose tolerance, and fatigue associated to sleep disordered breathing.

\section{Limitations of exercise tolerance in patients with idiopathic pulmonary fibrosis (IPF)}

Exercise limitation in patients with IPF has been attributed to mechanical constraint of ventilation and oxygen desaturation during cardiopulmonary exercise test (CPET) (24). In previous work, PH was found in $14 \%$ of patients with interstitial lung disease (ILD), and was associated to IPF presenting lower lung function parameters (25). Furthermore ventilation/perfusion imbalance has been described in those patients with complicated pulmonary hypertension $(\mathrm{PH})(26,27)$. Thus, these data emphasize the need for intensified treatment of IPF patients presenting PH. In the study presented by Chlumsky et al., the limits of exercise tolerance in IPF were investigated (28). In particular, they aimed to detect $\mathrm{PH}$ and test whether supplemental oxygen may improve exercise tolerance in those with significant oxygen desaturation. Fifty two patients with diagnosed IPF according to ATS/ERS criteria (VC $69 \pm 16 \%$ pred, TLCO $49 \pm 14 \%$ pred) were included in the study. $\mathrm{PeakVO}_{2}$ (\% predicted) showed positive correlation to tidal volume to vital capacity ratio $(\mathrm{VT} / \mathrm{VC})\left(\mathrm{pE} / \mathrm{VCO}_{2}\right.$ ratio at anaerobic threshold $(\mathrm{P}=0.005)$. The authors did not found any relationship between exercise tolerance and changes in $\mathrm{SpO}_{2} . \mathrm{PH}$ was associated with higher $\mathrm{VE} / \mathrm{VCO}_{2}$ ratio and lower transfer coefficient (KCO). Exercise tolerance in IPF seems to be limited mainly by mechanical constrain of ventilation and/or high dead space ventilation, while hypoxemia plays a minor role. Supplemental oxygen increased exercise tolerance, decreased physiological dead space and ventilatory demand during CPET, mainly in those with $\mathrm{PH}$. Exercise training has been proven to be a safe and effective behavioral intervention for prevention and rehabilitation of chronic conditions and recent results provide evidence of the safety and efficacy of exercise training intervention for IPF. However, while the benefits of exercise training in IPF patients have been described, the optimal program and limitations to exercise tolerance in these patients are still lacking. Therefore, the work presented by Chlumsky et al. elucidates that there are clear pathophysiological limitations in IPF patients that need to be taken into consideration when recommending supervised exercise training based pulmonary rehabilitation programs as the standard of care for IPF patients.

In conclusion, the studies presented highlight some translational aspects of current research on Clinical Physiology and Sleep, with a potential high impact in clinical practice. The physiological approach is essential to understand both the pathogenesis of clinical symptoms, like dyspnea, in respiratory diseases, and the complex interaction of control of breathing and sleep in different pathological conditions such as obesity, cancer and heart failure.

\section{Acknowledgements}

None.

\section{Footnote}

Conflicts of Interest: The authors have no conflicts of interest to declare.

\section{References}

1. Martinez-Garcia MA, Campos-Rodriguez F, Barbe F. Cancer and OSA: Current Evidence From Human Studies. Chest 2016;150:451-63.

2. Almendros I, Montserrat JM, Ramirez J, et al. Intermittent hypoxia enhances cancer progression in a mouse model of sleep apnoea. Eur Respir J 2012;39:215-7.

3. Nieto FJ, Peppard PE, Young T, et al. Sleep-disordered breathing and cancer mortality: results from the Wisconsin Sleep Cohort Study. Am J Respir Crit Care Med 2012;186:190-4.

4. Marshall NS, Wong KK, Cullen SR, et al. Sleep apnea and 20-year follow-up for all-cause mortality, stroke, and cancer incidence and mortality in the Busselton Health Study cohort. J Clin Sleep Med 2014;10:355-62.

5. Pataka A, Anttalainen U, Schiza S, et al. OSA and cancer in Europe: the European Sleep Apnea Database (ESADA) experience. Eur Respir J 2017;50:OA3209.

6. Martinez-Garcia MA, Campos-Rodriguez F, DuranCantolla J, et al. Obstructive sleep apnea is associated with cancer mortality in younger patients. Sleep Med 2014;15:742-8.

7. Campos-Rodriguez F, Martinez-Garcia MA, Martinez $M$, et al. Association between obstructive sleep apnea and cancer incidence in a large multicenter Spanish cohort. Am J Respir Crit Care Med 2013;187:99-105. 
8. Almendros I, Wang Y, Becker L, et al. Intermittent Hypoxiainduced Changes in Tumor-associated Macrophages and Tumor Malignancy in a Mouse Model of Sleep Apnea. Am J Respir Crit Care Med 2014;189:593-601.

9. Hakim F, Wang Y, Zhang SX, et al. Fragmented sleep accelerates tumor growth and progression through recruitment of tumor-associated macrophages and TLR4 signaling. Cancer Res 2014;74:1329-37.

10. Kukwa W, Migacz E, Druc K, et al. Obstructive sleep apnea and cancer: effects of intermittent hypoxia? Future Oncol 2015;11:3285-98.

11. Almendros I, Montserrat JM, Torres M, et al. Obesity and intermittent hypoxia increase tumor growth in a mouse model of sleep apnea. Sleep Med 2012;13:1254-60.

12. Obeso A, Gallego-Martin T, Gordillo A, et al. Aged mice obstructive sleep apnoea model with spontaneous tumorigenesis: physiological parameters. Eur Respir J 2017;50:OA1754.

13. Quintero M, Olea E, Conde SV, et al. Age protects from harmful effects produced by chronic intermittent hypoxia. J Physiol 2016;594:1773-90.

14. Kim HC, Mofarrahi M, Hussain SN. Skeletal muscle dysfunction in patients with chronic obstructive pulmonary disease. Int J Chron Obstruct Pulmon Dis 2008;3:637-58.

15. Donaldson AV, Maddocks M, Martolini D, et al. Muscle function in COPD: a complex interplay. Int J Chron Obstruct Pulmon Dis 2012;7:523-35.

16. Timmons JA, Knudsen S, Rankinen T, et al. Using molecular classification to predict gains in maximal aerobic capacity following endurance exercise training in humans. J Appl Physiol (1985) 2010;108:1487-96.

17. Rossiter H, Adami A, McDonal ML, et al. Genetic variants predicting the response to endurance exercise training are also associated with skeletal muscle oxidative capacity in COPD. Eur Respir J 2017;50:OA3428.

18. Sauleda J, Garcia-Palmer FJ, Tarraga S, et al. Skeletal muscle changes in patients with obstructive sleep apnoea

Cite this article as: Almendros I, Crespo A, Tura-Ceide O, Bonsignore MR. Clinical physiology and sleep: insights from the European Respiratory Society Congress 2017. J Thorac Dis 2017;9(Suppl 16):S1532-S1536. doi: 10.21037/jtd.2017.11.44 syndrome. Respir Med 2003;97:804-10.

19. Matsumoto T, Tanizawa R, Tachikawa R, et al. Impact of skeletal muscle in patients with obstructive sleep apnea. Am J Respir Crit Care Med 2017;195:A2622.

20. Opie GM, Catcheside PG, Usmani ZA, et al. Motor cortex plasticity induced by theta burst stimulation is impaired in patients with obstructive sleep apnoea. Eur J Neurosci 2013;37:1844-52.

21. Marillier M, Baillieul S, Le Roux Mallouf T, et al. Reduced voluntary activation and increased intracortical inhibition during leg extensions in severe obstructive sleep apnoea patients. Eur Respir J 2017;50:OA3432.

22. Yamamoto U, Mohri M, Shimada K, et al. Six-month aerobic exercise training ameliorates central sleep apnea in patients with chronic heart failure. J Card Fail 2007;13:825-9.

23. Ueno LM, Drager LF, Rodrigues AC, et al. Effects of exercise training in patients with chronic heart failure and sleep apnea. Sleep 2009;32:637-47.

24. Bonini M, Fiorenzano G. Exertional dyspnoea in interstitial lung diseases: the clinical utility of cardiopulmonary exercise testing. Eur Respir Rev 2017;26:160099.

25. Andersen CU, Mellemkjaer S, Hilberg O, et al. Pulmonary hypertension in interstitial lung disease: prevalence, prognosis and 6 min walk test. Respir Med 2012;106:875-82.

26. Collum SD, Amione-Guerra J, Cruz-Solbes AS, et al. Pulmonary Hypertension Associated with Idiopathic Pulmonary Fibrosis: Current and Future Perspectives. Can Respir J 2017;2017:1430350.

27. Andersen CU, Mellemkjaer S, Nielsen-Kudsk JE, et al. Pulmonary hypertension in chronic obstructive and interstitial lung diseases. Int J Cardiol 2013;168:1795-804.

28. Chlumsky J, Vasakova M, Sterclova M, et al. Limitations of exercise tolerance in patients with IPF. Eur Respir J 2017;50:OA1965. 